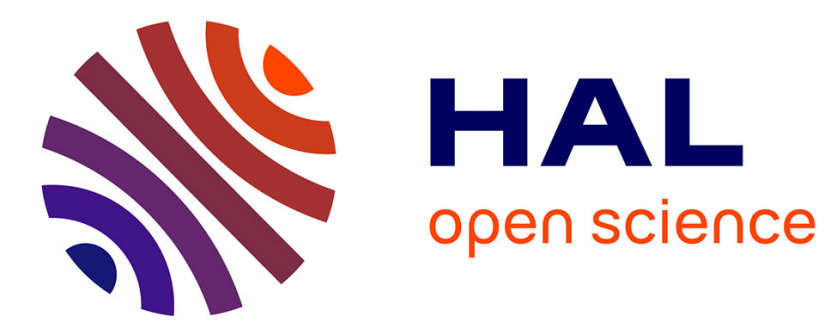

\title{
D-S Theory for Argument Confidence Assessment
}

Rui Wang, Jérémie Guiochet, Gilles Motet, Walter Schön

\section{To cite this version:}

Rui Wang, Jérémie Guiochet, Gilles Motet, Walter Schön. D-S Theory for Argument Confidence Assessment. 4th International Conference on Belief Functions (BELIEF 2016), Sep 2016, Prague, Czech Republic. pp.190-200, 10.1007/978-3-319-45559-4_20 . hal-01372016

\section{HAL Id: hal-01372016 https://hal.science/hal-01372016}

Submitted on 26 Sep 2016

HAL is a multi-disciplinary open access archive for the deposit and dissemination of scientific research documents, whether they are published or not. The documents may come from teaching and research institutions in France or abroad, or from public or private research centers.
L'archive ouverte pluridisciplinaire HAL, est destinée au dépôt et à la diffusion de documents scientifiques de niveau recherche, publiés ou non, émanant des établissements d'enseignement et de recherche français ou étrangers, des laboratoires publics ou privés. 


\title{
D-S theory for argument confidence assessment
}

\author{
Rui Wang ${ }^{1}$, Jérémie Guiochet ${ }^{1}$, Gilles Motet $^{1}$, and Walter Schön ${ }^{2}$ \\ 1 LAAS-CNRS, Université de Toulouse, CNRS, INSA,UPS, Toulouse, France \\ firstname.surname@laas.fr, \\ 2 Sorbonne Universités, Université de Technologie de Compiègne, Heudiasyc UMR CNRS 7253 CS \\ 60319 Compiègne Cedex France \\ wschon@utc.fr
}

\begin{abstract}
Structured arguments are commonly used to communicate to stakeholders that safety, security or other attributes of a system are achieved. Due to the growing complexity of systems, more uncertainties appear and the confidence in arguments tends to be less justifiable by reviewing. In this paper, we propose a quantitative method to assess the confidence in structured arguments, like safety cases. We adopt the Goal Structuring Notation (GSN) to model the safety case and propose to add annotations to identify uncertainties in this model. Three inference types of arguments are proposed according to their impact on confidence. Definition and quantification assessment of confidence are based on the belief function theory. The proposed approach is illustrated with several GSN examples.
\end{abstract}

Keywords: safety case, confidence assessment, belief function theory, assurance case

\section{Introduction}

Structured arguments play important role in communicating a system's attributes with various names: safety case [18, 2], assurance case [4], trust case [5], dependability case [3], etc. For safety-critical industries, such arguments are even required by the standards (ISO 26262 [15] for automotive, EN 50129 for railway, etc.).

Furthermore, regulation bodies (such as avionics certification authorities) have to evaluate the system safety based on safety cases in order to produce a justified decision for certification. Nevertheless, some problematic issues has been argued when assessing the structured arguments, especially for computing systems. In [6], authors consider that the excessive growth of argument leads it to be too complex to be analyzed. In $[1,14]$, the authors emphasize the necessity to assess the confidence in these arguments and propose to develop a confidence argument in parallel with the safety argument. Besides, some quantitative assessment of confidence in arguments are provided in [9] (using Bayesian Network), and [6,13] (using belief function theory for confidence definition).

This paper aims to propose a quantitative approach to assess the confidence in structured arguments. In order to give an understandable demonstration, a graphical notation, called Goal Structuring Notation (GSN) [18] presented in Section 2, is adopted to model the analyzed safety case including its identified uncertainties. Then, the quantified assessment process is developed under the frame of belief function theory in Section 3. Belief function theory allows uncertainty to be explicitly modeled and handled. An application of this approach is conducted for some extracts of GSN safety case in Section 4. 


\section{Safety argumentation}

Safety argument, also called safety case, is defined by [2] as "a documented body of evidence that provides a convincing and valid argument that a system is adequately safe for a given application in a given environment". It is used to communicate the rationale of developers for implementing the development or their choices of techniques. Many related research work are available based on the Toulmin's argument model [21]. [16] defined a notation of safety case, called Goal Structuring Notation (GSN), to make the presentation of argumentation more readable and adaptable. GSN allows the representation of the supporting evidence, objectives to be achieved, safety argument, context, etc. An example of GSN is given in Figure 1, which is derived from the Hazard Avoidance Pattern [17]. The five main elements of GSN are: goal (e.g., G1): the claim about the system; solution (e.g., Sn1): the reference to evidence item(s); strategy (e.g., S1): the nature of inference that exists between a goal and its supporting sub-goal(s); context (e.g., C1): a reference to contextual information, or a statement.

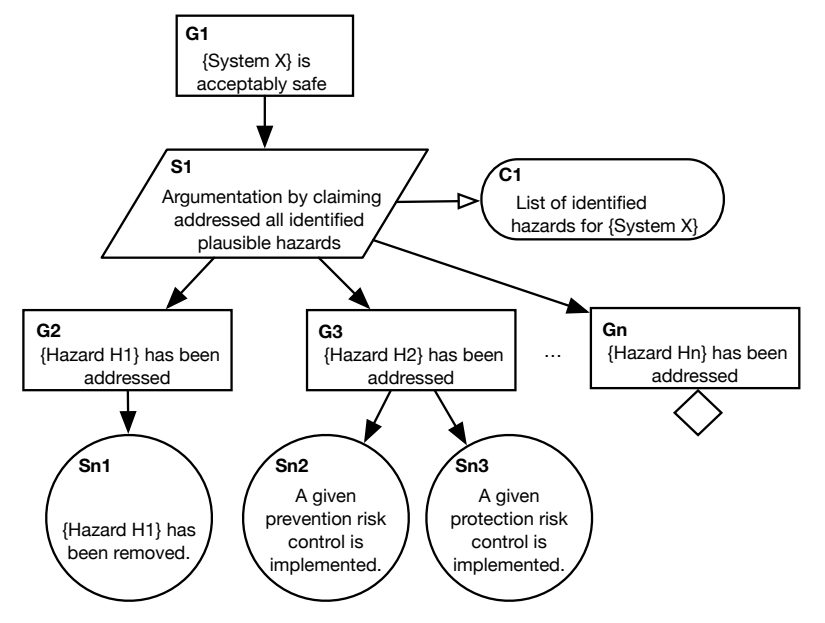

Fig. 1. GSN example adapted from Hazard Avoidance Pattern [17]

GSN provides a qualitative representation. Nevertheless, the confidence in the top claim is not specified in this view. Indeed, the correctness of the inference links, the appropriateness of the context, the sufficiency of the evidence are key factors to make the assertions in safety argument acceptable. However, in practice, no precise values can be assigned to these factors. We have to cope with uncertainties. We actually consider two types of uncertainties sources, which are similar to those presented in [14] named "appropriateness" and "trustworthiness". They are illustrated in the simple safety case presented in Figure 2 as uncertainty points (UPs): UP1 uncertainty in the fact that B supports A; and UP2 - uncertainty in the fact that B is True.

\section{Safety case confidence assessment}

This section presents the main contribution of the paper, which is a framework to quantitatively estimate the confidence in a safety case.

\subsection{Definition of confidence in argument}

We adopt the belief function theory, also called Dempster-Shafer theory (D-S theory) [7, 8, 20], to define the confidence in arguments. It is indeed one of the uncertainty theories with which 


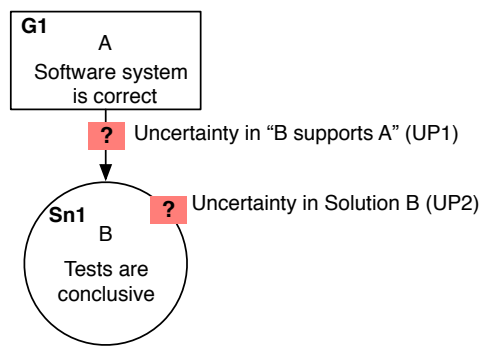

Fig. 2. Uncertainty points in a simple inference

you can explicitly estimate the uncertainty, and combine several sources of information which is particularly convenient to develop our confidence aggregation rules. For the claim $B$, ("Tests are conclusive") in Figure 2, the belief in its truth is expressed using the belief function and plausibility function provided in D-S theory. An opinion about this claim is assessed with 3 attributes: our belief $(\operatorname{bel}(\{B\}))$, our disbelief $(\operatorname{bel}(\{\bar{B}\}))$ in the conclusiveness of the testing, and the uncertainty $(\operatorname{pl}(\{B\})-\operatorname{bel}(\{B\}))$ concerning the fact that "we know that we don't know". This leads to have belief + disbelief + uncertainty $=1$.

In this paper, a binary frame of discernment $\Omega_{B}=\{\bar{B}, B\}$ is used to describe the truth of claim $B$; then we define the confidence in claim $B$ as follows:

$$
\left\{\begin{array}{l}
\operatorname{bel}(\{B\})=m^{\Omega_{B}}(\{B\})=g_{B} \\
\operatorname{bel}(\{\bar{B}\})=m^{\Omega_{B}}(\{\bar{B}\})=f_{B} \\
\operatorname{pl}(\{B\})-\operatorname{bel}(\{B\})=m^{\Omega_{B}}\left(\Omega_{B}\right)=1-m^{\Omega_{B}}(\{B\})-m^{\Omega_{B}}(\{\bar{B}\})=1-g_{B}-f_{B}
\end{array}\right.
$$

where $g_{P}, f_{P} \in[0,1]$.

Formalization of inferences (UP1) To study the inference between B and A, we propose to use a 2-tuple $\left(X_{B}, X_{A}\right)$ to express the cross product $\Omega_{B} \times \Omega_{A}$, where $X_{B}$ and $X_{A}$ are elements of $\Omega_{B}$ and $\Omega_{A}$ respectively. Then, the joint frame of discernment is $\Omega_{B} \times \Omega_{A}=$ $\{(\bar{B}, \bar{A}),(\bar{B}, A),(B, \bar{A}),(B, A)\}$. For instance, the mass describing the belief that claim $\mathrm{A}$ can be inferred from claim $\mathrm{B}$ and conversely that the fact that $\mathrm{A}$ is false can be inferred from the fact that $\mathrm{B}$ is false, is $m^{\Omega_{B} \times \Omega_{A}}(\{(B, A),(\bar{B}, \bar{A})\})$. This inference is measured by the contributing weight of $\mathrm{B}$ to $\mathrm{A}\left(w_{B}\right)$. Moreover, it is hard to ensure that the available supporting premises cover all aspects to assert the upper-level claim. Thus, we introduce a discounting factor $(v)$ to represent the completeness of premises. The contributing weight is defined as follows:

$$
m_{1}^{\Omega_{B} \times \Omega_{A}}(\{(\bar{B}, \bar{A}),(B, A)\})=w_{B} v \quad m_{1}^{\Omega_{B} \times \Omega_{A}}\left(\Omega_{B} \times \Omega_{A}\right)=1-w_{B} v
$$

Where $m_{1}^{\Omega_{B} \times \Omega_{A}}\left(\Omega_{B} \times \Omega_{A}\right)$ is used as abbreviation of $m_{1}^{\Omega_{B} \times \Omega_{A}}(\{(\bar{B}, \bar{A}),(\bar{B}, A),(B, \bar{A}),(B, A)\})$. When $v=1$, it means that B sufficiently support A; When $v=0, m_{1}^{\Omega_{B} \times \Omega_{A}}\left(\Omega_{B} \times \Omega_{A}\right)=1$ means that $\mathrm{B}$ does not provide any knowledge about $\mathrm{A}$, i.e. a full uncertainty exists in $\mathrm{A}$.

Formalization of the confidence in claims (UP2) The measure of confidence in B has been defined as Formula (1). In order to combine UP1 and UP2, we apply the vacuous extension [19] 
(represented by the up arrow $\uparrow$ ) to $m_{2}$, to transform the confidence in B on the frame $\Omega_{B}$ to the frame $\Omega_{B} \times \Omega_{A}$. Therefore, the confidence in claim B is:

$$
\left\{\begin{array}{l}
m_{2}^{\Omega_{B} \uparrow \Omega_{B} \times \Omega_{A}}\left(\{B\} \times \Omega_{A}\right)=g_{B} \\
m_{2}^{\Omega_{B} \uparrow \Omega_{B} \times \Omega_{A}}\left(\{\bar{B}\} \times \Omega_{A}\right)=f_{B} \\
m_{2}^{\Omega_{B} \uparrow \Omega_{B} \times \Omega_{A}}\left(\Omega_{B} \times \Omega_{A}\right)=1-f_{B}-g_{B}
\end{array}\right.
$$

Where $\{B\} \times \Omega_{A}$ is employed instead of $\{(B, A),(B, \bar{A})\}$ and $\{\bar{B}\} \times \Omega_{A}$ instead of $\{(\bar{B}, A),(\bar{B}, \bar{A})\}$ to highlight the ignorance in $\mathrm{A}$.

Confidence aggregation for simple inference Our aim is to deduce the degree of belief in the fact that claim A is true $(m(\{A\}))$ based on the belief placed in (2) and (3). With the help of Dempster's rule [20], these two pieces of information can be combined. The 6 possible combinations and focal sets are shown in Table 1, where the conflict factor K [20] in this combination rule is 0 , due to no conflict in this case. To calculate $m(\{A\})$ on $\Omega_{A}$ from the combined information on $\Omega_{B} \times \Omega_{A}$, we have to use the marginalization operation [19]. Accordingly, there is only one focal set $(\{(\mathrm{B}, \mathrm{A})\}$ underlined in Table 1$)$ contributing to $m(\{A\})$. Thus, the confidence in $\mathrm{A}$ is:

$$
\operatorname{bel}(\{A\})=m(A)=g_{B} w_{B} v
$$

where $g_{B}, w_{B}, v \in[0,1]$.

Table 1. Combination results of confidence measures

\begin{tabular}{|c|cc|}
\hline$m_{12}=m_{1} \oplus m_{2}$ & $m_{1}^{\Omega_{B} \times \Omega_{A}}(\{(\bar{B}, \bar{A}),(B, A)\})$ & $m_{1}^{\Omega_{B} \times \Omega_{A}}\left(\Omega_{B} \times \Omega_{A}\right)$ \\
\hline$m_{2}^{\Omega_{B} \uparrow \Omega_{B} \times \Omega_{A}}\left(\{B\} \times \Omega_{A}\right)$ & $\{(B, A)\}$ & $\{B\} \times \Omega_{A}$ \\
$m_{2}^{\Omega_{B} \uparrow \Omega_{B} \times \Omega_{A}}\left(\{\bar{B}\} \times \Omega_{A}\right)$ & $\{(\bar{B}, \bar{A})\}$ & $\{\bar{B}\} \times \Omega_{A}$ \\
$m_{2}^{\Omega_{B} \uparrow \Omega_{B} \times \Omega_{A}}\left(\Omega_{B} \times \Omega_{A}\right)$ & $\{(\bar{B}, \bar{A}),(B, A)\}$ & $\Omega_{B} \times \Omega_{A}$ \\
\hline
\end{tabular}

\subsection{Arguments inference types}

In practice, several premises are used to support one goal. Therefore, a new issue arises: how the contributions of premises are combined while assessing the confidence in a goal? Cyra and Gorski [6] extend the work of Govier [12] and introduce two inference rules, each of which contains 3 sub types of inferences. Nevertheless, there are overlapping among them. We propose another categorization of the argument with three inference types (listed in Table 2):

- Type 1: Dependent inference: premises supporting the same goal have some degree of dependency, denoted with $d$.

- $d=1$ : fully dependent inference. $\mathrm{B}$ and $\mathrm{C}$ are needed as premises of A. For instance, safety engineers often consider that a system is safe if the "test process is correct" as well as the "test results are correct". If one of these two premises is false, no confidence can be placed in the system safety. It is important to emphasize that this "dependence" means only that $\mathrm{A}$ depends on $\mathrm{B}$ and $\mathrm{C}$ (dependence formalized by joint mass function $m_{1}$ hereafter). The confidences in sub-claims B and C (formalized by mass functions $m_{2}$ and $m_{3}$ hereafter) are considered independent (there are independent elements of evidence in experts opinion) and can therefore be combined using Dempter's rule. In cases where this hypothesis would be not ensured, other combination rules like "cautious rule" [10] could be used instead. 
- $d=0.5$ : partial dependent inference. $\mathrm{B}$ and $\mathrm{C}$ have some impacts on each other when supporting A. For instance, safety engineers also consider that a system is safe if "highlevel requirement coverage is achieved", that is, all functions perform correctly, and "lowlevel requirement coverage is achieved", that is, all components are exercised. If we have high confidence in the function correctness, then we have high confidence in system safety. Then, even if we do not have sufficient evidence in the component exercising, we preserve our confidence in the system safety.

- Type 2: Redundant inference: premises contribute to the top goal with certain degree of redundancy, denoted with $r$.

- $r=1$ : fully redundant inference. B or $\mathrm{C}$ can be used alone as premises of A. For instance, safety engineers consider that "a failure of a hardware component is acceptable" (goal), if "its probability is low" or "the occurrence of this failure can be detected and handled" (fault tolerance mechanism). These two premises are fully redundant because if we have full confidence in one of them, then we believe that the goal is achieved.

- $r=0.5$ : partial redundant inference. B and $\mathrm{C}$ are not fully redundant, and can be used in a complementary way. For instance, safety engineers consider that "a system is acceptably safe" (goal), if "its test is conclusive" or "formal verification is passed". We believe that the two techniques (testing and formal verification) are partially redundant as they both partially contribute to our confidence in system safety.

- Type 3: Independent inference: each of the premises covers part of the conclusion, without redundancy or dependency $r / d=0$. For instance, safety engineers consider that confidence of system safety is obtained (goal), when all hazards are addressed. Therefore, the belief in each premise "Hazard Hi is addressed" provides an independent contribution to our confidence in system safety.

\subsection{Confidence assessment}

The proposed confidence aggregation operator varies according to the type of inference. Due to limited space, we only provide details for the dependent inference in this section. We assess the confidence in A based on the confidence in B and $\mathrm{C}$ and their way of contribution. The calculation is similar to Section 3.1, except that we need to take into account the argument types.

Formalization of inferences (UP1) In order to provide a general formula for the dependent inference, the cases with $d=0,0.5$ and 1 have to be taken into account. The independent contributions of $\mathrm{B}$ and $\mathrm{C}$ and the contribution of the combinations of $\mathrm{B}$ and $\mathrm{C}$ has to be considered. The corresponding mass function $m_{1}$ is therefore built using :

- A focal set giving the contribution of B to A (see Equation (3)), using the vacuous extension, to extend it to $\Omega=\Omega_{B} \times \Omega_{C} \times \Omega_{A}$,

- A focal set giving the contribution of $\mathrm{C}$ to A built on the same model and also extended to $\Omega$,

- A focal set giving the contribution of B and C to A (traduction of an AND operator in terms of mass function),

- The remaining mass being affected on $\Omega$.

Hence, this joint frame of discernment is expressed as $\Omega=\{(\bar{B}, \bar{C}, \bar{A}),(\bar{B}, \bar{C}, A),(\bar{B}, C, \bar{A}),(\bar{B}, C, A),(B, \bar{C}, \bar{A}),(B, \bar{C}, A),(B, C, \bar{A}),(B, C, A)\}$. According to D-S theory, the sum of masses of all the focal sets is 1 . We deduce that the degree of dependency $\left(d_{A}\right)$ is $1-w_{B}-w_{C}$. Also, we introduce the discounting factor $v$. Then, we obtain: 


$$
\left\{\begin{array}{l}
m_{1}^{\Omega}\left(\{\bar{B}\} \times \Omega_{C} \times\{\bar{A}\} \cup\{B\} \times \Omega_{C} \times\{A\}\right)=w_{B} v \\
m_{1}^{\Omega}\left(\Omega_{B} \times\{\bar{C}\} \times\{\bar{A}\} \cup \Omega_{B} \times\{C\} \times\{A\}\right)=w_{C} v \\
m_{1}^{\Omega}(\{(\bar{B}, \bar{C}, \bar{A}),(\bar{B}, C, \bar{A}),(B, \bar{C}, \bar{A}),(B, C, A)\})=d_{A}=\left(1-w_{B}-w_{C}\right) v \\
m_{1}^{\Omega}(\Omega)=1-v
\end{array}\right.
$$

where $v, w_{B}, w_{C} \in[0,1]$, and $w_{B}+w_{C} \leq 1$.

Formalization of the confidence in claims (UP2) According to the definition of confidence in Formula (1) of Section 3.1 and the help of vacuous extension, the measures of confidence in sub-claims B and $\mathrm{C}$ are:

$$
\begin{gathered}
\left\{\begin{array}{l}
m_{2}^{\Omega}\left(\{B\} \times \Omega_{C} \times \Omega_{A}\right)=g_{B} \\
m_{2}^{\Omega}\left(\{\bar{B}\} \times \Omega_{C} \times \Omega_{A}\right)=f_{B} \\
m_{2}^{\Omega}(\Omega)=1-f_{B}-g_{B}
\end{array}\right. \\
\left\{\begin{array}{l}
m_{3}^{\Omega}\left(\Omega_{B} \times\{C\} \times \Omega_{A}\right)=g_{C} \\
m_{3}^{\Omega}\left(\Omega_{B} \times\{\bar{C}\} \times \Omega_{A}\right)=f_{C} \\
m_{3}^{\Omega}(\Omega)=1-f_{C}-g_{C}
\end{array}\right.
\end{gathered}
$$

Confidence aggregation for dependent argument (Type 1) As explained above, mass functions $m_{1}, m_{2}$ and $m_{3}$ are considered as independent pieces of evidence and can therefore legitimately combined using Dempster's rule. According to the associativity of this rule, (6) and (7) are combined firstly, then with (5). Due to the limited scale of this paper, the development is not presented here. Thus, we give the confidence in claim A:

$$
\operatorname{bel}(\{A\})=m(\{A\})=v\left(\left(1-w_{B}-w_{C}\right) g_{B} g_{C}+g_{B} w_{B}+g_{C} w_{C}\right)
$$

Formula (8) is the confidence aggregation formula for the basic dependent argument for two premises. The general confidence aggregation formula for n-nodes dependent argument derived:

$$
m(\{A\})=v\left[\left(1-\sum_{i=1}^{n} w_{i}\right) \prod_{i=1}^{n} g_{i}+\sum_{i=1}^{n} g_{i} w_{i}\right]
$$

Where $n>1, g_{i}, w_{i}, v \in[0,1]$, and $\sum_{i=1}^{n} w_{i} \leq 1$.

Confidence aggregation for redundant argument (Type 2) For redundant argument, the confidence aggregation operator has to be changed. The redundant parts of the premises behave as an OR gate. Therefore, the contributing weights are measured in the following way:

$$
\left\{\begin{array}{l}
m_{1}^{\Omega}\left(\{\bar{B}\} \times \Omega_{C} \times\{\bar{A}\} \cup\{B\} \times \Omega_{C} \times\{A\}\right)=w_{B} v \\
m_{1}^{\Omega}\left(\Omega_{B} \times\{\bar{C}\} \times\{\bar{A}\} \cup \Omega_{B} \times\{C\} \times\{A\}\right)=w_{C} v \\
m_{1}^{\Omega}(\{(\bar{B}, \bar{C}, \bar{A}),(\bar{B}, C, A),(B, \bar{C}, A),(B, C, A)\})=r_{A}=\left(1-w_{B}-w_{C}\right) v \\
m_{1}^{\Omega}(\Omega)=1-v
\end{array}\right.
$$

Then the developing process is similar to the one used for the dependent argument. We directly give the confidence aggregation formula for the above argument: 


$$
m(\{A\})=v\left(\left(1-w_{B}-w_{C}\right)\left[1-\left(1-g_{B}\right)\left(1-g_{C}\right)\right]+g_{B} w_{B}+g_{C} w_{C}\right)
$$

The general formula for n-node redundant argument is deduced. We obtain:

$$
m(\{A\})=v\left(\left(1-\sum_{i=1}^{n} w_{i}\right)\left[1-\prod_{i=1}^{n}\left(1-g_{i}\right)\right]+\sum_{i=1}^{n} g_{i} w_{i}\right)
$$

Where $n>1, g_{i}, w_{i}, v \in[0,1]$, and $\sum_{i=1}^{n} w_{i} \leq 1$.

\section{Experimental application}

In this section, the confidence aggregation formulas are applied to five simple examples with two premises. This application aims to analyze how the confidence evolves depending on different inference types. The examples in Table 2 are extractions of GSN patterns from [22] or objectives required in the avionics standard DO-178C [11].

The formulas corresponding to varied values of $d$ or $r$ are obtained based on Equation (8) and (11). Note that when the premises are completely independent, i.e. $r / d=1-w_{B}-w_{C}=0$, then Equation (8) and (11) are equivalent.

Moreover, we use the contour plotting to illustrate the behaviors of these five cases with several values for $d$ and $r$, as shown in Table 2. As $v$ is the common factor of all formulas, its impact on the confidence in A is easily estimated. We choose $v=1$ for this analysis.

In the figures representing the behaviors, red means low confidence in A. On the contrary, green represents high confidence. Comparing the positions of Point $\mathrm{M}\left(g_{B}=0, g_{C}=1\right)$ and Point $\mathrm{N}\left(g_{B}=1, g_{C}=0\right)$, the best model, with which it is easy to obtain high confidence, is the fully redundant case (case 1); the opposite is case 5 , that is, the fully dependent inference. Looking at cases 2 and 4 , the obvious difference is that low confidence in one single premise of partial dependent inference decreases more confidence in A than that of partial redundant inference. Furthermore, this feature helps to determine the right type of inference for an argument. In cases 2, 3 and 4, because the weight of B is higher than the weight of C, the influence of $g_{B}$ on $\operatorname{bel}(A)$ is always larger than $g_{C}$ (Point $\mathrm{M}$ is always higher than Point $\mathrm{N}$ ).

According to this analysis, the behaviors of the aggregation formulas are consistent with our expectation regarding the confidence variation. The different impacts of contributing weights make explicit the influence of the dependency and redundancy among arguments on the confidence in the top goal.

\section{Conclusion}

In this paper, we propose a quantitative approach to evaluate the confidence in safety arguments. A graphical safety notation, GSN, is used to clearly present the studied arguments. The definition and aggregation of confidence for simple inference and multiple inferences are all realized by adopting the functions and operations of the belief function theory. We introduce a clear way to categorize three inference types including five cases. This makes it possible to explicitly assess the contributions of premises. We applied our approach to typical examples of arguments in system safety engineering domain, and checked that results of aggregation rules are consistent. A behavior analysis is given to demonstrate the characters of the proposed aggregation formulas. A case study of real system safety case will be our future work. Experts will be inquired to provide the reasonable confidence values with the help of the GSN examples. The issue of decision-making based on confidence levels (see behavior figures in Table 2) will be also considered. 
Table 2. Experimental application of confidence aggregation formulas

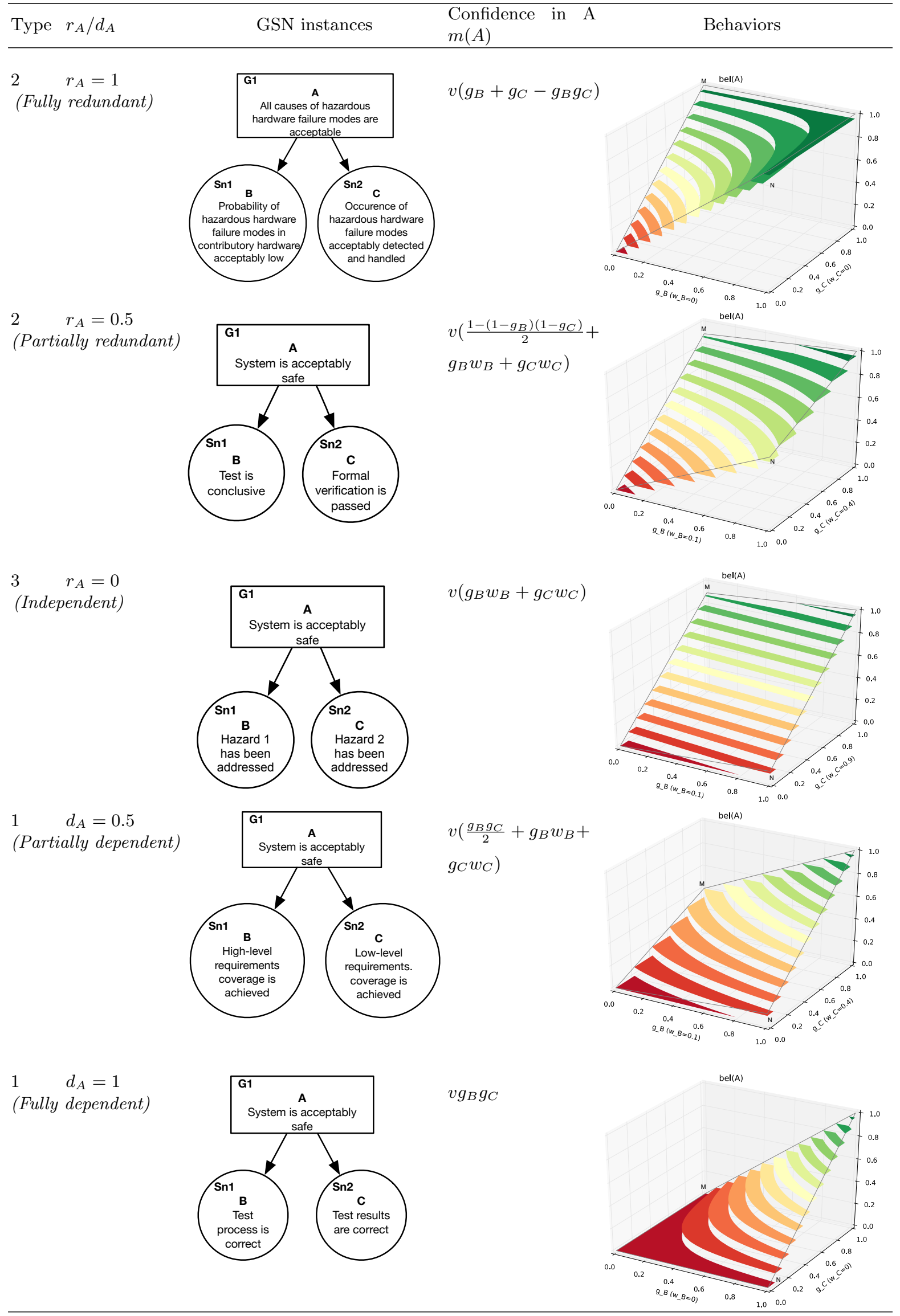




\section{References}

1. Anaheed Ayoub, BaekGyu Kim, Insup Lee, and Oleg Sokolsky. A systematic approach to justifying sufficient confidence in software safety arguments. In Computer Safety, Reliability, and Security, pages 305-316. Springer, 2012.

2. Peter Bishop and Robin Bloomfield. A methodology for safety case development. In Industrial Perspectives of Safety-Critical Systems, pages 194-203. Springer, 1998.

3. Robin Bloomfield, Bev Littlewood, and David Wright. Confidence: its role in dependability cases for risk assessment. In Dependable Systems and Networks, 200\%. DSN'0\%. 37th Annual IEEE/IFIP International Conference on, pages 338-346. IEEE, 2007.

4. Robin E Bloomfield, Sofia Guerra, Ann Miller, Marcelo Masera, and Charles B Weinstock. International working group on assurance cases (for security). Security ES Privacy, IEEE, 4(3):66-68, 2006.

5. Lukasz Cyra and Janusz Gorski. Supporting compliance with security standards by trust case templates. In Dependability of Computer Systems, 200\%. DepCoS-RELCOMEX'0\%. 2nd International Conference on, pages 91-98. IEEE, 2007.

6. Lukasz Cyra and Janusz Gorski. Support for argument structures review and assessment. Reliability Engineering ES System Safety, 96(1):26-37, 2011.

7. Arthur P Dempster. New methods for reasoning towards posterior distributions based on sample data. The Annals of Mathematical Statistics, pages 355-374, 1966.

8. Arthur P Dempster. Upper and lower probabilities induced by a multivalued mapping. The annals of mathematical statistics, pages 325-339, 1967.

9. Ewen Denney, Ganesh Pai, and Ibrahim Habli. Towards measurement of confidence in safety cases. In Empirical Software Engineering and Measurement (ESEM), 2011 International Symposium on, pages 380-383. IEEE, 2011.

10. Thierry Denœux. The cautious rule of combination for belief functions and some extensions. In 2006 9th International Conference on Information Fusion, pages 1-8. IEEE, 2006.

11. DO-178C/ED-12C. Software considerations in airborne systems and equipment certification, 2011. RTCA/EUROCAE.

12. Trudy Govier. A practical study of argument. Wadsworth, Cengage Learning, 2013.

13. Jérémie Guiochet, Quynh Anh Do Hoang, and Mohamed Kaaniche. A model for safety case confidence assessment. In Computer Safety, Reliability, and Security (SAFECOMP), pages 313-327. Springer, 2015.

14. Richard Hawkins, Tim Kelly, John Knight, and Patrick Graydon. A new approach to creating clear safety arguments. In Advances in systems safety, pages 3-23. Springer, 2011.

15. ISO 26262. Software considerations in airborne systems and equipment certification, 2011. International Organization for Standardization (ISO).

16. Tim Kelly. Arguing Safety - A Systematic Approach to Safety Case Management. PhD thesis, Department of Computer Science, University of York, 1998.

17. Tim Kelly and John McDermid. Safety case construction and reuse using patterns. In Computer Safety, Reliability, and Security (SAFECOMP), pages 55-69. Springer, 1997.

18. Tim Kelly and Rob Weaver. The goal structuring notation-a safety argument notation. In Proceedings of the Dependable Systems and Networks (DSN) workshop on assurance cases, 2004.

19. David Mercier, Benjamin Quost, and Thierry Denœux. Contextual discounting of belief functions. In Symbolic and Quantitative Approaches to Reasoning with Uncertainty, pages 552-562. Springer, 2005.

20. Glenn Shafer. A mathematical theory of evidence, volume 1. Princeton university press Princeton, 1976.

21. Stephen E Toulmin. The uses of argument. Cambridge University Press, 2003.

22. Robert Andrew Weaver. The safety of software: Constructing and assuring arguments. PhD thesis, Department of Computer Science, University of York, 2003. 